\title{
AIRS retrieval validation during the EAQUATE
}

\author{
Daniel K. Zhou*a, William L. Smith ${ }^{\text {b,c }}$, Vincenzo Cuomo ${ }^{\mathrm{d}}$, Jonathan P. Taylor ${ }^{\mathrm{e}}$, Christopher D. \\ Barnet $^{\mathrm{f}}$, Paolo Di Girolamo ${ }^{\mathrm{g}}$, Gelsomina Pappalardo ${ }^{\mathrm{d}}$, Allen M. Larar ${ }^{\mathrm{a}}$, Xu Liu ${ }^{\mathrm{a}}$, Stuart M. Newman ${ }^{\mathrm{e}}$, \\ Clare Lee ${ }^{\mathrm{e}}$, and Stephen A. Mango ${ }^{\mathrm{h}}$ \\ ${ }^{a}$ NASA Langley Research Center, Hampton, VA, USA \\ ${ }^{\mathrm{b}}$ Hampton University, Hampton, VA USA \\ ${ }^{c}$ University of Wisconsin-Madison, Madison, WI, USA \\ ${ }^{\mathrm{d}}$ Istituto di Metodologie per l'Analisi Ambientale - Consiglio Nazionale delle Ricerche, Tito Scalo (Potenza), IT \\ ${ }^{\mathrm{e} U K}$ Met Office, Exeter, Devon, UK \\ ${ }^{\mathrm{f}} \mathrm{NOAA} / \mathrm{NESDIS}$, Camp Springs, MD, USA \\ ${ }^{\mathrm{g}}$ DIFA, Università degli Studi della Basilicata, Potenza, IT \\ ${ }^{\text {h}}$ NPOESS Integrated Program Office, Silver Spring, MD, USA
}

\begin{abstract}
Atmospheric and surface thermodynamic parameters retrieved with advanced hyperspectral remote sensors of Earth observing satellites are critical for weather prediction and scientific research. The retrieval algorithms and retrieved parameters from satellite sounders must be validated to demonstrate the capability and accuracy of both observation and data processing systems. The European AQUA Thermodynamic Experiment (EAQUATE) was conducted mainly for validation of the Atmospheric InfraRed Sounder (AIRS) on the AQUA satellite, but also for assessment of validation systems of both ground-based and aircraft-based instruments which will be used for other satellite systems such as the Infrared Atmospheric Sounding Interferometer (IASI) on the European MetOp satellite, the Cross-track Infrared Sounder (CrIS) from the NPOESS Preparatory Project and the following NPOESS series of satellites. Detailed inter-comparisons were conducted and presented using different retrieval methodologies: measurements from airborne ultraspectral Fourier transform spectrometers, aircraft in-situ instruments, dedicated dropsondes and radiosondes, and ground based Raman Lidar, as well as from the European Center for Medium range Weather Forecasting (ECMWF) modeled thermal structures. The results of this study not only illustrate the quality of the measurements and retrieval products but also demonstrate the capability of these validation systems which are put in place to validate current and future hyperspectral sounding instruments and their scientific products.
\end{abstract}

Keywords: Remote sensing, retrieval, validation, AIRS, NAST-I, ARIES, LIDAR.

\section{INTRODUCTION}

The Atmospheric InfraRed Sounder (AIRS) on the Earth Observing System (EOS) Aqua satellite was launched on 4 May 2002. The AIRS sounding goals are 1K RMS temperature in 1-km layer averages and $15 \%$ RMS moisture in 2-km layer averages; the specifications are found elsewhere ${ }^{1}$. A great deal of calibration and validation activity has been done to understand the instrument and to quantify its data products ${ }^{2-4}$. The AIRS Science Team has developed a retrieval scheme to produce atmospheric and surface properties. Validation of retrieved temperature and moisture profiles has been made through comparisons with the European Center for Medium range Weather Forecasting (ECMWF, see website $\mathrm{http}: / / \mathrm{www}$.ecmwf.int/ for more information) model forecast and match up radiosonde measurements ${ }^{1,3-4}$. In the mean time, the NPOESS Airborne Sounder Testbed - Interferometer (NAST-I) developed by the National Polar-orbiting Operational Environmental Satellite System (NPOESS) Integrated Program Office (IPO) and the Scanning-High resolution Interferometer Sounder (S-HIS) of the University of Wisconsin - Madison ${ }^{5}$ flown on high altitude aircraft, such as the ER-2 and Proteus, have gone through numerous field campaigns ${ }^{6}$. These instruments provide experimental

*Daniel.K.Zhou@NASA.GOV; phone: 1-757-864-5663; fax: 1-757-864-7790 
observations needed for finalizing specifications and testing proposed designs and data processing algorithms for the Cross-track Infrared Sounder (CrIS) which will fly on NPOESS. Detailed descriptions of NAST-I instrumentation, data processing methodologies, and data products can be found elsewhere ${ }^{7-11}$. Selected AIRS radiance data sets have gone through the NAST-Team retrieval algorithm since AIRS data first became available in July $2002^{12}$. Through these validation and evaluation activities, the AIRS retrieval algorithm has been greatly improved over the last few years, producing more accurate retrievals.

Aircraft under flights of Aqua have been providing observations for both radiance and retrieval evaluation ${ }^{6,12-13}$. An international experiment, EAQUATE (European Aqua Thermodynamic Experiment), was held during September 2004 in Italy and the United Kingdom to demonstrate certain ground-based and airborne systems useful for validating hyperspectral sounding observations from satellites during this decade and the next. The focus of this initial experiment was placed on validation of the AIRS instrument on the EOS Aqua satellite ${ }^{14-15}$. A great deal of effort has been given to the data collected during EAQUATE. In this paper, we report the validation results of the thermodynamic parameters, the atmospheric temperature and moisture profiles. The inter-comparison efforts have been made in a few different perspectives: (1) retrieval algorithm inter-comparison through their retrieval products, (2) retrieval inter-comparison between two instruments (i.e., AIRS and NAST-I) using the same retrieval algorithm, (3) profile retrieval validation through comparisons with radiosonde, dropsonde, and Raman Lidar measurements, and (4) atmospheric structure intercomparison with that displayed by the ECMWF model analysis. It is worthy to point out that the ECMWF model analysis is partially influenced by multi-sensor observations and/or assimilated instrumental data including AIRS and radiosondes. We conclude by comparing the various results of this validation activity, which illustrate the quality of the measurements and retrieval products, as well as the capability of the validation systems used.

\section{RETRIEVAL ALGORITHMS}

It is equally important to validate the retrievals and the retrieval algorithm producing the geophysical products. In this study, one of the major objectives is to validate retrieval algorithms applied to AIRS data. Two retrieval algorithms are used here for inter-comparison; they are described in detail elsewhere ${ }^{1,10,16}$. However, the differences and similarities of these retrieval algorithms are addressed here in brief. Retrievals from NAST-I and AIRS using the same retrieval methodology (i.e., called the NAST-Team retrieval methodology, hereafter denoted as N-T) are compared with AIRS retrievals obtained by the latest version (Version 4.0) of the AIRS Science Team retrieval methodology (hereafter denoted as A-T). Table 1 summarizes the major differences and similarities of these two retrieval algorithms.

Table 1. Major Similarities and Differences between A-T and N-T Retrieval Methodologies

\begin{tabular}{|c|c|c|c|}
\hline & A-T AIRS/AMSU & N-T AIRS & N-T NAST-I \\
\hline Forward Model & SARTA & SARTA & OSS \\
\hline Reg. Ret. Methodology & EOF & EOF & EOF \\
\hline Reg. Training profile & Global ECMWF & Regional Radiosondes & Regional Radiosondes \\
\hline Reg. Training Radiance & AIRS Measured (clear) & SARTA Simulated & OSS Simulated \\
\hline Reg. Channel \# & 1688 & 1526 & 4424 \\
\hline Phy. Ret. Methodology & Regularization & Regularization & Regularization \\
\hline Phy. Ret. Procedure & Sequential & Simultaneous & Simultaneous \\
\hline Phy. Ret. Channel \# & 156 & 575 & 697 \\
\hline Dealing with Clouds & Cloud Clearing with AMSU & Ret. to Cloud Top & Ret. to Cloud Top \\
\hline Surface Emissivity Ret. & Reg. followed by Phy. & EOF Reg. & EOF Reg. \\
\hline
\end{tabular}

Note: Regression denoted as Reg., Physical denoted as Phy., and Retrieval denoted as Ret.

The N-T retrieval methodology uses eigenvector regression to obtain initial profiles ${ }^{10,17-18}$. The radiance eigenvectors are generated from radiances calculated using a forward radiative transfer model from a regional and seasonal climatology of radiosonde data. For NAST-I retrievals, 4424 spectral channels are used; whereas for AIRS, 1526 spectral channels are used in N-T retrievals. The N-T retrieval uses only infrared radiance data from NAST-I or AIRS within a retrieval procedure that directly accounts for the influence of clouds on the observed radiances, but the retrieval validity is generally restricted to above cloud top level. The regression result is used as a first guess to a matrix inverse solution of the radiative transfer equation. In the physical matrix inverse retrieval step, the N-T uses an iterative 
simultaneous matrix inverse solution for all variables based on a selection of 697 NAST-I channels and 575 AIRS channels for the NAST-I and AIRS physical retrievals, respectively. Surface and cloud spectral emissivity is determined by EOF (i.e., Empirical Orthogonal Function) regression and used in the matrix inverse solution. The N-T retrieval algorithm is applied to both AIRS and NAST-I data to minimize the impact of retrieval algorithm differences on the retrieval products. However, it should be noted that the forward radiative transfer models used differ in that the Optimal Spectral Sampling (OSS) fast molecular radiative transfer model ${ }^{19-20}$ is used for the NAST-I retrieval while the Standalone AIRS Radiative Transfer Algorithm (SARTA) ${ }^{21}$ is used for the AIRS retrieval.

The A-T retrieval methodology uses eigenvector regression to obtain initial profiles as well ${ }^{1,16,22}$. The A-T regression is based on a global database of profiles extracted from ECMWF analyses co-located with actual AIRS cloud-cleared radiances using 1688 AIRS spectral channels. In the case of the A-T retrievals, microwave data from the Advanced Microwave Sounding Unit (AMSU) instrument ${ }^{1}$ aboard the Aqua satellite are used to cloud clear the radiance data and provide additional sounding radiance information for retrieval in a clouded atmosphere. The regression coefficients are generated, relating the atmospheric state variables to the radiance eigenvector amplitudes. In the physical retrieval, the A-T uses a sequential approach and a total of 156 AIRS spectral channels in which 65 spectral channels are used for temperature, 42 for water vapor, 26 ozone, and 23 for surface temperature. The retrieval of surface emissivity is specified using synthetic radiance regression relationships, similar to the N-T retrieval and followed by a physical approach $^{16}$. The SARTA is used for the A-T AIRS retrieval.

\section{VALIDATION ON THERMODYNAMICS PRODUCTS}

There were two phases of the EAQUATE campaign: one was performed in Italy and the other in the United Kingdom. The Proteus aircraft had 4 flights passing over the ground station (Potenza) many times where numerous ground-based instruments were operational ${ }^{15}$. However, the ground-based measurements from Potenza used in this work are from Vaisala-type radiosondes and two Raman Lidar systems ${ }^{23-25}$. One Raman Lidar station (labeled Lidar \#1) is located at Potenza (40³9'N latitude, $15^{\circ} 48^{\prime}$ E longitude, $730 \mathrm{~m}$ above sea level), while another Raman Lidar station (labeled Lidar \#2) and a radiosonde station are located at Tito Scalo, Potenza (40³6’ $\mathrm{N}$ latitude, $15^{\circ} 44^{\prime} \mathrm{E}$ longitude, $760 \mathrm{~m}$ above sea level). Lidar \#1 measures both water vapor and temperature, while Lidar \#2 measures only water vapor. For this latter system, relative humidity $(\mathrm{RH})$ is computed with a coincident radiosonde temperature. Two Proteus flights dedicated to AIRS validation flown over Potenza were scheduled when the Aqua satellite was also passing over Potenza during the nights of 7-8 and 9-10 September 2004. During the second phase over the United Kingdom, the Proteus aircraft, together with the UK's Facility for Airborne Atmospheric Measurements (FAAM) BAe146 aircraft ${ }^{14}$, made two dedicated flights for AIRS validation during the days of both 14 and 18 September 2004. Numerous in-situ sensors and remote sensing instruments flew on BAe146 aircraft, and dropsondes were released from the BAe146 during the experiments. The wealth of data from ground-based and aircraft, in-situ and remote sensing instruments, were collected during this experiment to capture accurately the atmospheric state observed by the satellite instruments during the same period. These data have been used for detailed investigation, evaluation, and validation in this study; however, only a fraction of data is presented herein to demonstrate and conclude the results for this work.

\subsection{7-8 September over Potenza, Italy}

The Aqua satellite passed over Potenza at 01:08 UTC on 8 September 2004. The Proteus aircraft passed over a few times, with the one overpass was made at 01:10 UTC being close to the Aqua satellite overpass. Radiosondes were launched from Potenza at 23:00 UTC on 7 September and 02:00 UTC on 8 September. Both Raman Lidar systems were operational. Raman Lidar data were processed using a 10-minute integration time and variable vertical averaging in order to reach a higher altitude for retrieval validation as Aqua and Proteus were passing over. During that time, the sky was generally clear, with a few spots of low scattered clouds. The temperature, water vapor mixing ratio, and relative humidity profiles from the Potenza area are shown in Figure 1. AIRS retrievals from both A-T and N-T retrieval algorithms use the same cloud cleared radiance produced by the AIRS team. The A-T cloud clearing procedure depends on AMSU measurements. Therefore, the AIRS spatial resolution has to be degraded to the AMSU spatial resolution, about $3 \times 3$ AIRS single field of view (FOV) and having a diameter of $45 \mathrm{~km}$ at nadir. All NAST-I retrievals from NAST-I single FOV falling into AIRS $3 \times 3$ FOV (or AMSU FOV) are averaged, the time being when Proteus flew over Potenza at about 02:00 UTC on 8 September. The ECMWF model analyses were interpolated to AIRS FOV location 
and time. Two radiosonde observations ( 23:00 UTC and $\sim 02: 00$ UTC) from Potenza were averaged. Raman Lidar data were extensively processed for the Aqua satellite overpass at 01:08 UTC and for the Proteus aircraft overpass at 02:15 UTC; the averaged profiles of these two overpasses are plotted in Figure 1. The profile differences (deviation from the A-T AIRS retrieval) are also shown on the right panels. As shown, A-T AIRS temperature deviates slightly from other profiles as a group except that A-T and N-T AIRS temperature profiles agree with each other below an altitude of $5 \mathrm{~km}$. Differences between N-T NAST and N-T AIRS retrievals are expected from differences in NAST and AIRS instrument FOV, spectral resolution, and noise level. NAST-I retrievals for this case are matched more closely, than the AIRS retrievals, to the measurements of radiosonde and Raman Lidar, as well as the analysis of ECMWF model. As for the comparison of water vapor mixing ratio and relative humidity profiles, two Raman Lidar profiles indicate some difference, especially in the region near $3 \mathrm{~km}$ where few scattered clouds were observed. A large difference between Raman Lidar and other profiles in that region is due to the difference of vertical resolution; the Raman Lidar has a higher signal to noise ratio and the vertical resolution is about 15-30 m. AIRS water vapor mixing ratio and RH profiles retrieved with A-T and N-T algorithms agree well, except in the region near $9 \mathrm{~km}$. In that region, the radiosonde indicates a lower humidity than that indicated by all other sensors. The NAST-I retrieval compares well with the radiosonde in the altitudes below $8 \mathrm{~km}$. The NAST-I and N-T AIRS retrievals agree well except in the region below $3 \mathrm{~km}$, which could be a result of the difference in instrument spatial resolution. In general, these temperature and relative humidity profiles compare favorably to one another. After carefully considering the particulars addressed above, as a single profile comparison for this case, the data agree within the accuracy of the retrievals claimed for the AIRS and NAST-I instruments.
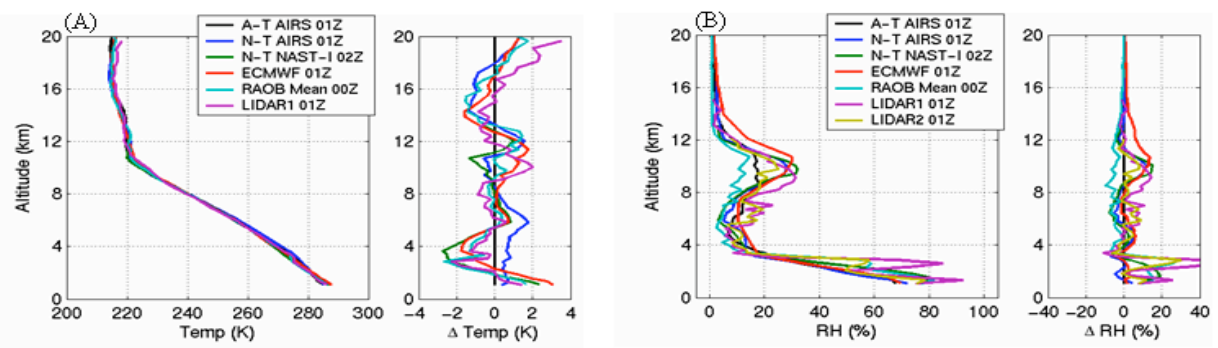

Figure 1. Inter-comparison of (A) temperature and (B) relative humidity profiles from Potenza during the night of 7-8 September 2004 (see text). Radiosonde and Lidar data are linear interpolated into AIRS and/or NAST retrieval grid.

The temperature and relative humidity cross sections along the Aqua ground track (i.e., AIRS nadir viewing) are illustrated in Figure 2. The cross section of the temperature deviation from the mean reveals the details of temperature spatial variation. The general patterns (i.e., the spatial features) shown in the ECMWF model analysis compare favorably with AIRS retrievals. However, the profile discrepancy between ECMWF and AIRS retrieval can be much greater than the required AIRS retrieval accuracy from location to location due to the difference in vertical resolution and horizontal resolution. Detailed thermodynamic structure of atmosphere is captured by AIRS measurements in both A-T and N-T retrievals. There is excellent correspondence between the spatial features revealed by these cross-sections, despite the differences between the two retrieval algorithms. It is noted, however, that slightly larger spatial gradients result with the N-T algorithm. As shown in Figure 3, the cross section of retrieval difference between N-T and A-T reveals a small but detailed structure. A standard deviation (STD) and a mean derived from the cross section of retrieval difference are plotted in the left panel. For temperature, the standard deviation error (i.e., STDE) profile is about 0.5-1.0 $\mathrm{K}$ and the mean difference (i.e., bias) is within $\pm 1.0 \mathrm{~K}$. For relative humidity, the standard deviation profile is less than $10 \%$ at altitudes above $3 \mathrm{~km}$, but it exceeds $10 \%$ in the terrestrial boundary layer (TBL). The mean difference profile is within $\pm 8 \%$. The statistical results are within the requirements of this type of sounder. These small differences can be explained by the different vertical resolution achieved with the different retrieval algorithms, which is also shown in the profile validation over Potenza (Figure 1). A relatively large difference in relative humidity near the surface is mostly from land observations, possibly a result of the difference in the manner in which surface emissivity is handled in the two different retrieval schemes. A relatively large difference in surface skin temperature, shown in panel C of Figure 3, could be initiated by an error of the land surface emissivity in the retrieval. The error in the surface emissivity (or skin temperature) resulted in a large moisture error near the surface to compensate for land surface uncertainty. For example, a large skin temperature difference existing over land (e.g., latitude less than $33^{\circ} \mathrm{N}$, and greater than $50^{\circ} \mathrm{N}$ ) is associated with the large relative humidity difference in the TBL shown in Figure 3. As seen in the figure, a large skin temperature 
difference between two retrievals accrues in the vicinity of the Sahara Desert (in this case, in the region of latitude of $33^{\circ} \mathrm{N}$ or less). In that area the surface emissivity retrieved from two retrieval schemes is quite different. In general, A-T retrieved skin temperature is cooler than N-T while A-T emissivity is higher than N-T. Accurately retrieving land surface properties is still a challenge and further studies are needed to improve retrieval methodology for land surface conditions.

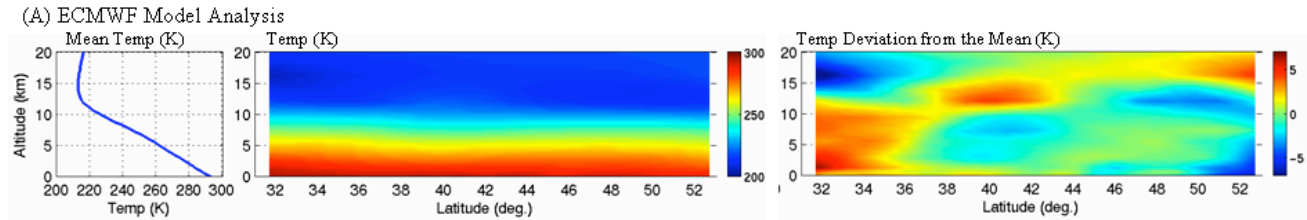

(B) A-T Retrieval from Cloud Cleared Radiance

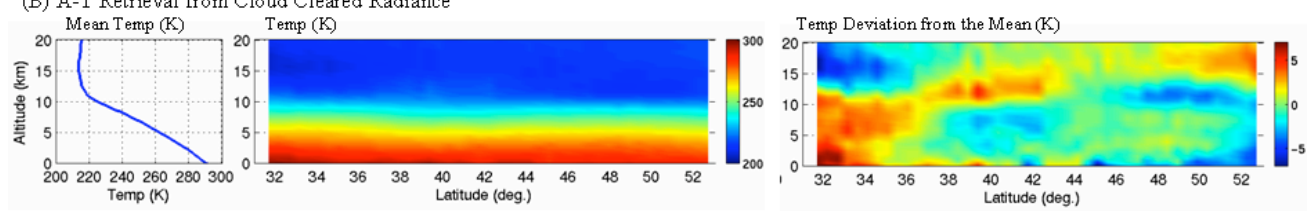

(C) N-T Retrieval from Cloud Cleared Radiance

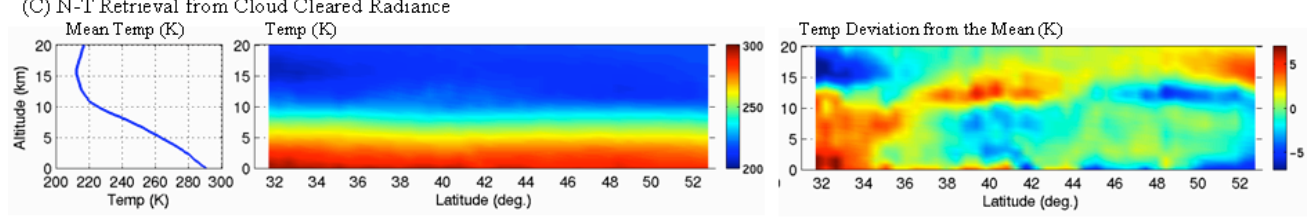

(D) ECMWF Model Analysis

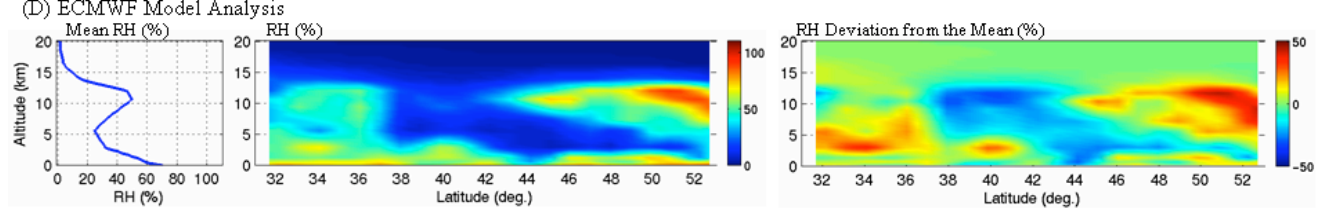

(E) A-T Retrieval from Cloud Cleared Radiance

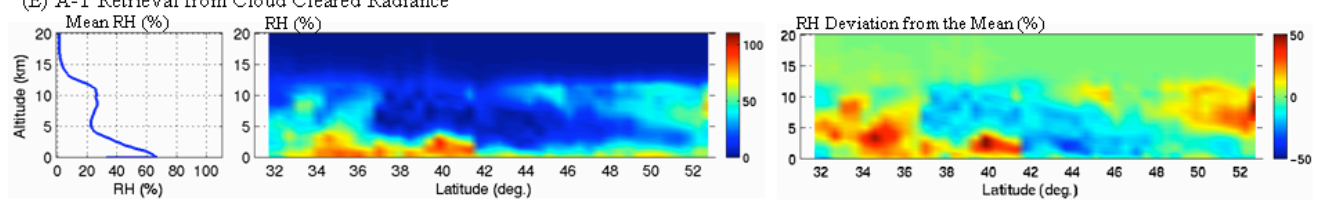

(F) N-T Retrieval from Cloud Cleared Radiance

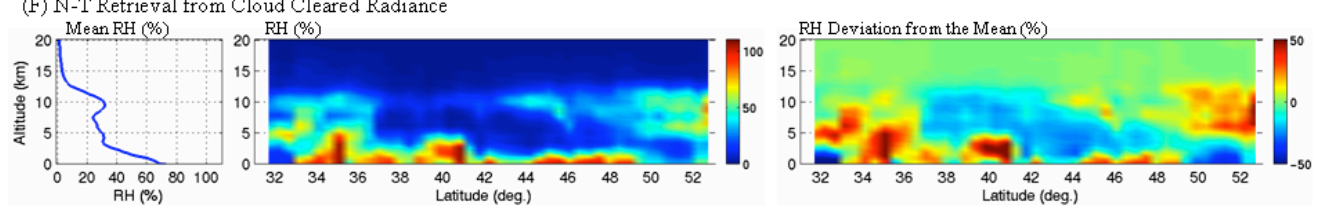

Figure 2. Temperature cross section of AIRS granule at nadir: ECMWF model analyses, A-T retrievals, and N-T retrievals are in (A), (B), and (C), respectively. Relative humidity cross section of ECMWF model analyses, A-T retrievals, and N-T retrievals are in $(\mathrm{D}),(\mathrm{E})$, and $(\mathrm{F})$, respectively. The cross section mean profile and the deviation from the mean are plotted (see text).

Inter-comparison between AIRS and NAST-I has been conducted with N-T retrieval algorithm using AIRS original single FOV data. NAST-I retrievals were spatially degraded to AIRS single FOV footprints for inter-comparison. ECMWF model analyses were also interpolated to AIRS single FOV footprints. Figure 4, panels A and B, shows the effective skin temperature retrieved from AIRS and NAST-I with a full spatial resolution of $\sim 15 \mathrm{~km}$ and $\sim 2 \mathrm{~km}$, respectively. In the figure, the lines with arrows indicate the Aqua and Proteus flight directions with the associated times. The solid/open circles represent the AIRS single field of view (FOV) within the NAST-I ground track swath width. The cloudy regions are indicated with dashed open circles. AIRS, NAST-I, and ECMWF mean temperature and relative humidity profiles of the section (indicated with the open circles) are plotted in panels C and D with a Vaisalatype radiosonde and two Raman Lidar observations from Potenza. It is worth pointing out that (1) the averaged profile 
reduced vertical resolution of a single FOV retrieval has a lower vertical resolution while radiosonde and Raman Lidar observations have a much higher vertical resolution and (2) a dry bias in the humidity measurement from a Vaisala-type radiosonde at altitudes above $\sim 8 \mathrm{~km}$ has been noticed here as well as in other validations. The temperature and relative humidity cross sections and the deviation from their means are plotted in Figure 4. In spite of the cloudy regions (e.g., $\sim 38.2^{\circ} \mathrm{N}$ Latitude and $40-41^{\circ} \mathrm{N}$ Latitude), the atmospheric features are captured by both AIRS and NAST-I sounders as well as the ECMWF modeled structure. Small differences between AIRS and NAST-I are noticed due to the observation time differences between AIRS and NAST-I. The fine vertical structures (i.e., resolution) of the retrieved profiles are partially due to the instrumentation differences such as the spectral resolution and instrument noise, which could cause a difference in the retrieved profiles. Overall, the retrievals from the two different sounders compare favorably with each other. The difference of the section means is plotted within the uncertainty of retrieved products. This difference implies that both instruments are well calibrated and the two radiative transfer models used are both accurate. The advantage of using an aircraft remote sounder, like NAST-I, is that continuous spectrum of atmospheric radiation is obtained with a high spatial resolution as needed to accurately validate the satellite observations.

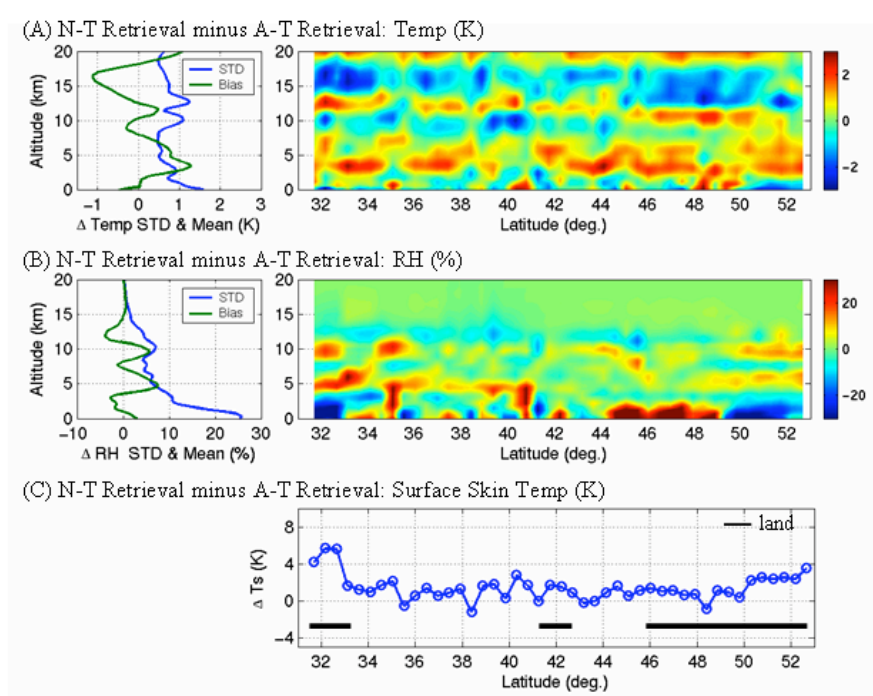

Figure 3. The cross sections of (A) temperature difference and (B) relative humidity difference between A-T and N-T algorithms. Left panels plot a standard deviation (in blue) and a mean bias (in green) of the retrieval difference. The difference of surface skin temperature is plotted (C) and the black bars indicate the measurements over the land.

\subsection{4 and 18 September over Celtic Sea, United Kingdom}

Similar analyses have been conducted for two AIRS granule data sets when Proteus and BAe146 were flown under Aqua on 14 and 18 September 2004. Proteus and BAe146 were flying at approximately the same location and time for these two dates. A series of 5 and 9 dropsondes were released from BAe146 in a close location and time on 14 and 18 September, respectively ${ }^{14}$. Figure 5 shows the different atmospheric conditions from day to day captured by the measurements. The top panels show the A-T effective surface skin temperature retrieved from AIRS cloud cleared radiance. It is noticed that clouds affecting actual skin temperature retrievals were present in the sky and this is reflected in the data. However, the sky was almost clear at the locations where Proteus (flight track indicated in magenta) and BAe146 aircraft were flying. The inter-comparisons for temperature, water mixing ratio, and relative humidity profiles were made at AIRS $3 \times 3 \mathrm{FOV}$ (or AMSU FOV) located at $50.84^{\circ} \mathrm{N}$ latitude and $6.68^{\circ} \mathrm{W}$ longitude on 14 September, and at $50.99^{\circ} \mathrm{N}$ latitude and $6.85^{\circ} \mathrm{W}$ longitude on $18 \mathrm{September}$, respectively. The available nearby radiosondes launched at the stations marked with open triangles on the top panels were used in the profile comparison. The dropsondes from BAe146 were dropped during the same period when NAST-I was taking the measurements. The approximate time of the profile (or profile mean) is indicated in the figure legend. Overall, the agreement between these profiles (i.e., A-T AIRS, N-T AIRS, NAST-I, ECMWF, Radiosonde, and Dropsondes) is equivalent to that shown for Potenza, Italy. The difference is within what is expected of instrumental limitations and retrieval uncertainties. However, there is a vertical feature in the temperature profile of 14 September for the tropopause region, 10-16 km. A somewhat larger variation in 
NAST-I and N-T AIRS retrievals is pronounced; this variation is also shown in the radiosonde profile, but not as pronounced as in the retrievals. A relatively large temperature discrepancy in this region was also indicated from other data sets (e.g., shown in Figure 4), but less pronounced. Other detailed evaluations between the different algorithms, different instrumental measurements, and ECMWF model analyses, have been performed with these two AIRS granules. Results similar to what was shown early for the Italian granule were obtained.
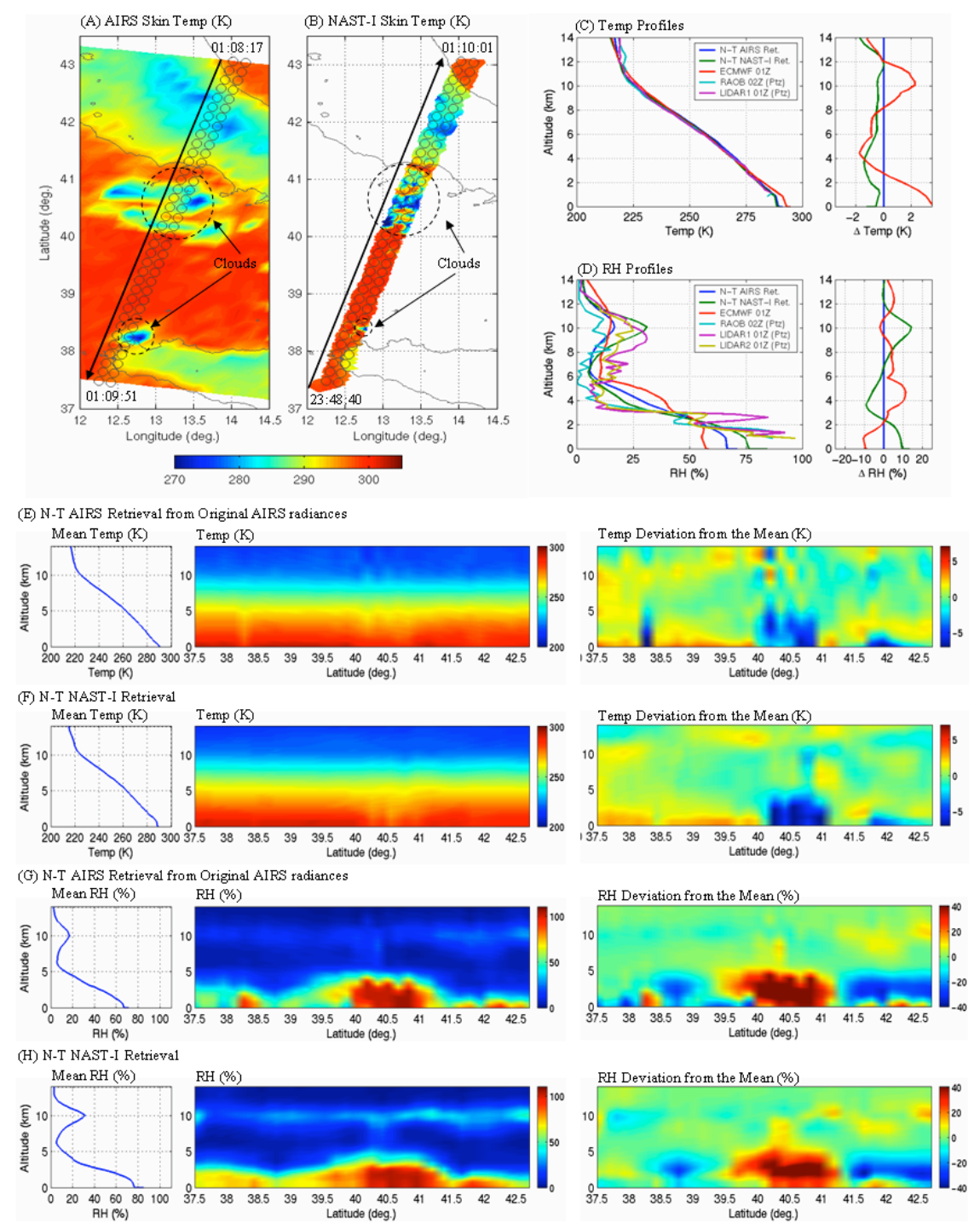

Figure 4. (A) and (B) plot AIRS and NAST-I retrieved effective surface skin temperature, respectively, during the night of 7-8 September 2004. Section mean profile of (C) temperature and (D) relative humidity using AIRS and NAST-I data processed through N-T retrieval scheme. Radiosonde and Raman Lidar profiles from Potenza, as well as section mean ECMWF model analysis are plotted as references. Temperature cross sections of A-T retrievals and N-T retrievals are in (E) and (F), respectively. Relative humidity cross sections of A-T retrievals and N-T retrievals are in $(\mathrm{G})$ and $(\mathrm{H})$, respectively. The cross section mean profile and the deviation from the mean are plotted (see text).

Since these two cases were made over the sea with the BAe146 aircraft flown as low as $30 \mathrm{~m}$, the surface properties, like the sea surface temperature (SST) and emissivity, are observed with the Airborne Research Interferometer Evaluation System (ARIES) interferometer on board ${ }^{26}$. The Proteus and BAe146 aircraft flew under the Aqua satellite within AIRS nadir observation on 14 September and AIRS $31.22^{\circ}$ off nadir on 18 September 2004. NAST-I and ARIES observations within the AIRS FOV are used for inter-comparisons. The NAST-I viewing angle is limited to near nadir (less than $15^{\circ}$ ) 
and the ARIES nadir observations. The geophysical locations of observations are illustrated in Figure 6, the AIRS and ECMWF data are interpolated to the mean location of ARIES. The AIRS SST is contributed from a fairly large area in comparison with NAST-I and ARIES observations. AIRS SST retrieved with A-T and N-T retrieval algorithms, the mean SST of NAST-I retrievals, and of ARIES retrievals are listed in Table 2 as well as ECMWF analyses. The geophysical locations and FOVs are illustrated in Figure 6. ARIES SST is a little warmer than AIRS and NAST-I retrievals by a mean of $0.37 \mathrm{~K}$. Retrieved SSTs are cooler than ECMWF SST, which may be related to the bulk temperatures reported by the floating buoys. The SSTs retrieved from AIRS, NAST-I, and ARIES radiance are expected to be cooler than the sea surface bulk temperature due to evaporative cooling ${ }^{27-28}$. A bias (normally within a few tenths of a degree) existing between infrared sensed and in-situ measured sea surface temperature is the physical difference between sea surface skin temperature and in-situ sea surface temperature measured at some depth ${ }^{28}$. Results of AIRS SST from these two cases revealed here are consistent with what was reported by Aumann et al. ${ }^{29}$. Their associated surface emissivity spectra are plotted in Figure 6 against laboratory measured seawater emissivity ${ }^{30}$. As seen, the ARIES emissivity agrees very well with laboratory measured seawater emissivity for both cases. The emissivity spectra derived from NAST-I and ARIES are nearly unchanged from day-to-day. Despite the difference of the AIRS viewing angle (i.e., satellite zenith angle) and wind speed from 14 September to 18 September, the seawater emissivity spectra derived from different instruments, platforms, viewing geometry, and retrieval algorithms are in fair agreement for these cases. Small differences, such as ARIES emissivity and laboratory measured seawater emissivity being slightly higher (i.e., $\sim 0.006-$ $0.010)$ than AIRS and NAST-I, are shown. The retrieved seawater emissivity is relatively good as a diverse surface database was used in the retrieval to handle different surface types. A compensation for the emissivity difference may explain why the SST of AIRS and NAST-I are slightly lower $(\sim 0.35 \mathrm{~K})$ than ARIES. Nevertheless, these differences are within the expectation of these instruments (i.e., AIRS and NAST-I) and their data processing procedures because atmospheric effects or contributions are included in the retrieval schemes.
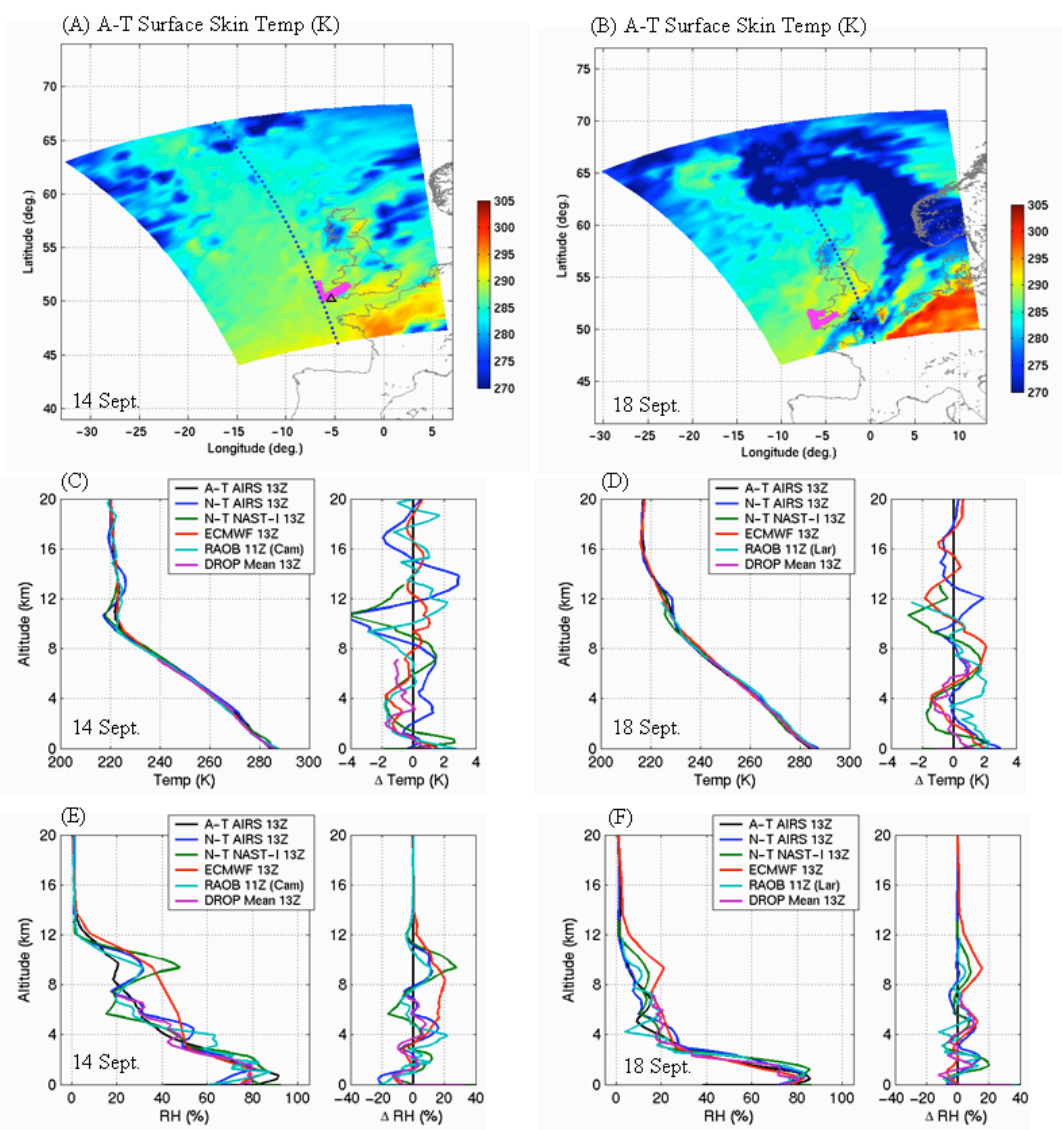

Figure 5. Effective skin temperatures of AIRS granules (A and B) are plotted for 14 and 18 September 2004, respectively. The temperature $(\mathrm{C}$ and $\mathrm{D})$ and relative humidity (E and F) profile inter-comparisons are plotted for 14 and 18 September 2004, respectively (see text). 

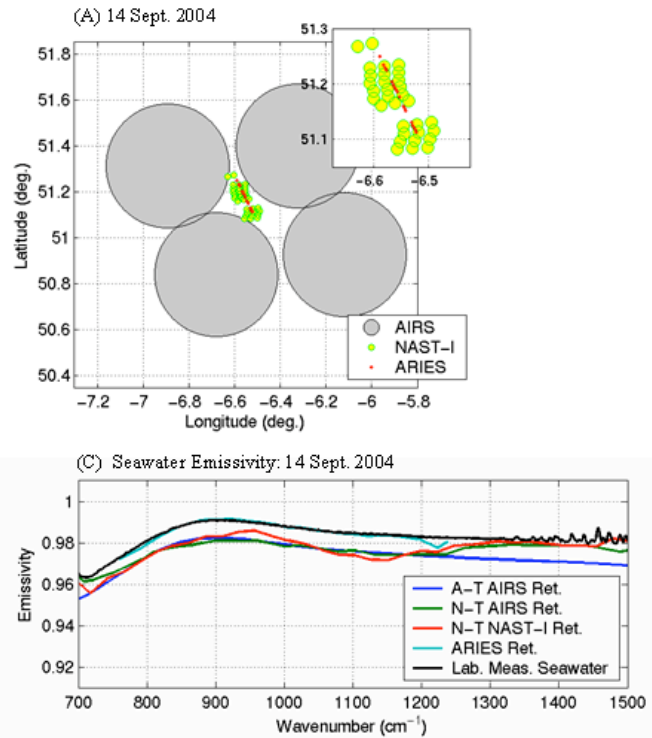
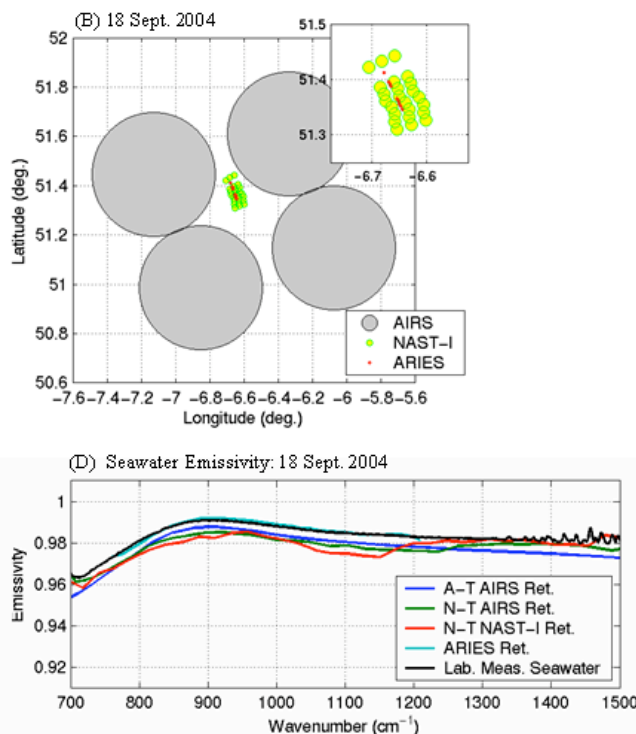

Figure 6. Geophysical locations and approximate FOVs from AIRS, NAST-I, and ARIES used for surface properties intercomparison are plotted. Large gray circles are AIRS $3 \times 3$ single FOVs equivalent to AMSU FOV. Four AIRS/AMSU FOVs are used to interpolate to the value centered at ARIES' observations. The footprints of NAST-I, and ARIES are enlarged at the top-right. Cases for 14 and 18 September 2004 are plotted in (A) and (B) respectively. Seawater emissivity inter-comparisons between AIRS retrievals from A-T and N-T, NAST-I retrieval, ARIES retrieval, and laboratory measurement: 14 and 18 September 2004 measurements are plotted in (C) and (D), respectively (see text).

Table 2. SST Inter-comparison

\begin{tabular}{|c|c|c|c|c|c|c|c|c|}
\hline & \multicolumn{4}{|c|}{14 September 2004} & \multicolumn{4}{|c|}{18 September 2004} \\
\hline & Lat. (N) & Lon. (W) & SST (K) & $\Delta \mathrm{SST}(\mathrm{K})^{*}$ & Lat. (N) & Lon. (W) & $\mathrm{SST}(\mathrm{K})$ & $\Delta \mathrm{SST}(\mathrm{K})^{*}$ \\
\hline A-T AIRS/Aqua & 51.18 & 6.56 & 287.91 & -0.37 & 51.37 & 6.66 & 287.81 & -0.35 \\
\hline N-T AIRS/Aqua & 51.18 & 6.56 & 287.94 & -0.34 & 51.37 & 6.66 & 287.82 & -0.34 \\
\hline NAST-I/Proteus & 51.17 & 6.56 & 287.96 & -0.32 & 51.37 & 6.65 & 287.66 & -0.50 \\
\hline ARIES/BAe146 & 51.17 & 6.55 & 288.28 & +0.00 & 51.37 & 6.66 & 288.16 & +0.00 \\
\hline ECMWF & 51.18 & 6.56 & 289.04 & +0.76 & 51.37 & 6.66 & 288.61 & +0.45 \\
\hline
\end{tabular}

*SST minus ARIES retrieved SST

\section{SUMMARY}

The international experiment, EAQUATE, was successful in testing ground-based and airborne systems for validating hyperspectral satellite measurements. High quality data were collected during the experiment. These data are used for AIRS data validation as demonstrated in this paper. This study demonstrates the need for both high density in-situ observations for detailed accurate validation and high altitude aircraft sounders like the NAST-I or SHIS in order to provide instant radiance and retrieval validation. High altitude aircraft remote sounders provide broad area coverage with high spatial resolution and continuous spectral coverage as needed to validate satellite observations.

Retrieval validation studies have been conducted in a manner which enables the effects of retrieval algorithm accuracy and satellite measurement accuracy to be separated. A variety of independent validation systems, such as radiosondes, dropsondes, ground-based Raman Lidar, and the ECMWF model analysis are included. Several conclusions are made from this study. (1) Very similar atmospheric spatial structure was retrieved from AIRS and NAST-I observatrions. (2) Good agreement is obtained between the retrieval products (i.e., AIRS and NAST-I), and radiosondes, dropsondes, and Raman Lidar. (3) General retrieval product agreement is obtained for two different retrieval algorithms, one the AIRS 
team and the other, the NAST team algorithm. The agreement is approximately within $1 \mathrm{~K}$ for temperature STD and $10 \%$ for relative humidity STD above the TBL, and $10-25 \%$ in the TBL. (4) AIRS surface skin temperature retrieved from the two retrieval algorithms has an offset which is greatly pronounced over the land. This surface skin temperature deference is due to the manner in which surface emissivity is handled by the two different algorithms. The retrieval uncertainties in the surface properties (i.e., emissivity and temperature) affect the accuracy of the thermodynamic profiles in the TBL. (5) Many validation cases over Potenza with Proteus and Aqua overpasses having dedicated radiosonde and Raman Lidar observations reveal that there is a potentially dry bias in Vaisala-type radiosonde observations in an altitude range of $8-11 \mathrm{~km}$; this bias is revealed from the inter-comparisons between radiosonde, AIRS retrievals, NAST-I retrievals, and two Raman Lidar observations. (6) The ECMWF analysis has a relatively poor spatial resolution (in both vertical and horizontal) in comparison with AIRS data. The profile discrepancy between ECMWF and AIRS retrieval can be much greater than that required to validate AIRS retrieval accuracy. However, the spatial features shown in the ECMWF model analysis compare favorably with AIRS retrievals. (7) All the temperature and moisture sounding retrievals are in generally good agreement with each other and the coincident radiosonde, dropsonde, and Raman Lidar observations. (8) The surface properties over water (i.e., SST and emissivity) are retrieved within expected accuracy and compared favorably with observations from all instruments from the variety of platforms. The overall agreement indicates that the latest version (i.e., Version 4.0) of the archived AIRS/AMSU retrievals is reasonably accurate, at least for clear sky oceanic conditions. This experiment has demonstrated the use of ground-based and aircraft-based validation for current and future hyperspectral satellite sounders. This work has also established the need for validating retrieval algorithms to ensure that the same retrievals can be produced from alternative retrieval algorithms.

\section{ACKNOWLEDGEMENTS}

The authors greatly appreciate the contributions of the NASA Langley Research Center, the Space Science and Engineering Center of the University of Wisconsin - Madison, the NASA Jet Propulsion Laboratory-led AIRS science team, the Istituto di Metodologie per l'Analisi Ambientale - Consiglio Nazionale delle Ricerche, the Università degli Studi della Basilicata, and the UK Met Office. AIRS data used in this study were provided by the NASA Goddard Space Flight Center and the NOAA National Environmental Satellite Data and Information Service. The NAST-I program is supported by the NPOESS IPO, NASA Headquarters, and NASA Langley Research Center. The FAAM is jointly

funded by the UK Met Office and the Natural Environment Research Council. The personnel who contributed to this study are too numerous to mention by name, nonetheless their personal contributions are greatly appreciated.

\section{REFERENCES}

1. J. Susskind, C. Barnet, J. Blaisdell, L. Iredell, F. Keita, L. Kouvaris, G. Molnar, and M. T. Chahine: "Accuracy of geophysical parameters derived from Atmospheric Infrared Sounder/Advanced Microwave Sounding Unit as a function of fractional cloud cover", J. Geophys. Res., 111, doi:10.1029/2005JD006272 (2006).

2. H. H. Aumann, and coauthors, "AIRS/AMSU/HSB on the Aqua mission: design, science objective, data products, and processing systems", IEEE Trans. Geosci. Remote Sensing, 41, 253-264 (2003).

3. D. C. Tobin, and coauthors: "Atmospheric Radiation Measurement site atmospheric state best estimates for Atmospheric Infrared Sounder temperature and water retrieval validation", J. Geophys. Res., 111, D09S14 doi:10.1029/2005JD006103 (2006).

4. M. G. Divakarla, C. D. Barnet, M. D. Goldberg, L. M. McMillin, E. Maddy, W. W. Wolf, L. Zhou, and X. Liu: "Validation of Atmospheric Infrared Sounder temperature and water vapor retrievals with matched radiosonde measurements and forecasts", J. Geophys. Res., 111, D09S15doi:10.1029/2005JD006116 (2006).

5. H. E. Revercomb, and co-authors, "Recent results from two new aircraft-based Fourier-transform interferometers: The Scanning High-resolution Interferometer Sounder and the NPOESS Atmospheric Sounder Testbed Interferometer", Proc. $8^{\text {th }}$ Int. Workshop on Atmospheric Science from Space using Fourier Transform Spectrometry (ASSFTS), Toulouse, France (1998).

6. D. C. Tobin, and coauthors: "Radiometric and spectral validation of Atmospheric Infrared Sounder observations with the aircraft-based Scanning High-Resolution Interferometer Sounder",J. Geophys. Res., 111, D09S02 doi:10.1029/2005JD006094 (2006). 
7. D. Cousins, and W. L. Smith, "National Polar-Orbiting Operational Environmental Satellite System (NPOESS) Airborne Sounder Testbed-Interferometer (NAST-I)", Proc. SPIE, 3127, 323-331 (1997).

8. W. L. Smith, A. M. Larar, D. K. Zhou, C. A. Sisko, J. Li, B. Huang, H. B. Howell, H. E. Revercomb, D. Cousins, M. J. Gazarik, D. Mooney, "NAST-I: results from revolutionary aircraft sounding spectrometer", Proc. SPIE, 3756, 2-8 (1999).

9. A. M. Larar, W. L. Smith, and D. K. Zhou, "Spectral radiance validation studies using NAST-I and other independent measurement systems", Proc. SPIE, 4485, 81-90 (2002).

10. D. K. Zhou, D. K., W. L. Smith, J. Li, H. B. Howell, G. W. Cantwell, A. M. Larar, R. O. Knuteson, D. C. Tobin, H. E. Revercomb, and S. A. Mango, "Thermodynamic product retrieval methodology for NAST-I and validation", Applied Optics, 41, 6957-6967 (2002).

11. W. L. Smith, D. K. Zhou, A. M. Larar, S. A. Mango, H. B. Howell, R. O. Knuteson, H. E, Revercomb, and W. L. Smith Jr., "The NPOESS Airborne Sounding Testbed Interferometer - remotely sensed surface and atmospheric conditions during CLAMS”, J. Atmos. Sci., 62, 1117-1133 (2005).

12. W. L. Smith, D. K. Zhou, H. E. Revercomb, H. L. Huang, P. Antonelli, and S. A. Mango, "Hyperspectral atmospheric sounding", Proc. SPIE 5235, 389-396 (2004).

13. M. M. Gunshor, D. Tobin, T. J. Schmit, and W. P. Menzel, "First satellite intercalibration comparing high spectral resolution AIRS with operational geostationary imagers", $12^{\text {th }}$ Conference on Satellite Meteorology and Oceaongraphy, Longbeach, CA, 9-13 February 2003 (preprints). Boston, MA, American Meteorological Society (2003).

14. W. Smith, J. Taylor, V. Cuomo, A. Larar, P. Antonelli, S. Mango, C. Serio, D. Zhou, and G. Pappalardo, "EAQUATE - An international experiment for hyper-spectral atmospheric sounding validation", in Fourier Transform Spectroscopy/Hyperspectral Imaging and Sounding of the Environment Topical Meeting on CD-ROM (the Optical Society of America, Washington, DC, 2005), JMA4 (2005).

15. V. Cuomo, and coauthors, "The Italian phase of the EAQUATE measurement campaign", Proc. SPIE 5979, 396409 (2005).

16. J. Susskind, C. D. Barnet, and J. M. Blaisdell, "Retrieval of atmospheric and surface parameters from AIRS/AMSU/HSB data in presence of clouds", IEEE Trans. Geosci. Remote Sensing, 41, 390-409 (2003).

17. D. K. Zhou, W. L. Smith, and A. M. Larar, "Surface temperature and emissivity from airborne measurements of IR radiance spectra", Eos Trans. AGU, 82(47) (2001).

18. D. H. DeSlover, R. O. Knuteson, B. Osborne, D. K. Zhou, and W. L. Smith, "Validation of aircraft-measured land surface emissivity", Proc. SPIE, 4891, 384-391, 2003.

19. J. L. Moncet, and Coauthors, "Algorithm theoretical basis document (ATBD) for the Cross Track Infrared Sounder (CrIS) environmental data records (EDR)", V1.2.3, AER Document Number P882-TR-E-1.2.3-ATBD-03-01 (2003).

20. X. Liu, J.-L. Moncet, D. K. Zhou, and W. L. Smith, "A Fast and Accurate Forward Model for NAST-I Instrument", in Fourier Transform Spectroscopy and Optical Remote Sensing of Atmosphere, 2003 Technical Digest Series OSA (Optical Society of America, Washington, D.C., 2003), p. 16 (2003).

21. L. L. Strow, S. E. Hannon, S. De Souza-Machado, H. E. Motteler, and D. Tobin, "An overview of the AIRS radiative transfer model”, IEEE Trans. Geosci. Remote Sensing, 41, 303-313 (2003).

22. M. D. Goldberg, Y. Qu., L. M. McMillin, W. Wolf, L. Zhou, and M. Divakarla, "AIRS near real-time products and algorithms in support of operational numerical weather prediction", IEEE Trans. Geosci. Remote Sensing, 41, 379389 (2003).

23. G. Pappalardo, A. Amodeo, S. Amoruso, L. Mona, M. Pandolfi, V. Cuomo, "One year of tropospheric Lidar measurements of aerosol extinction and backscatter", Ann. Geophys., 46, 401-413 (2003).

24. P. Di Girolamo, R. Marchese, D. N. Whiteman, and B. B. Demoz, "Rotational Raman Lidar measurements of atmospheric temperature in the UV", Geophys. Res. Lett., 31, L01106, doi:10.1029/2003GL018342 (2004).

25. P. Di Girolamo, A. Behrendt, and V. Wulfmeyer, "Pure rotational Raman lidar measurements of atmospheric temperature and extinction from space: performance simulations", App. Opt. 45, 2474-2494 (2006).

26. S. M. Newman, J. A. Smith, M. D. Glew, S. M. Rogers, and J. P. Taylor, "Temperature and salinity dependence of sea surface emissivity in the thermal infrared", Q. J. Royal Meteorol. Soc., 131, 2539-2557 (2005).

27. P. Schluessel, H. Y. Shin, W. J. Emery, and H. Grassl, "Comparison of satellite-derived sea surface temperatures with in situ skin measurements", J. Geophys. Res., 92, 2895-2874 (1987).

28. W. L. Smith, R. O. Knuteson, H. E. Revercomb, W. Feltz, H. B. Howell, W. P. Menzel, N. R. Nalli, O. Brown, J. Brown, P. Minnett, and W. McKeown, "Observations of the infrared radiative properties of the ocean - implications 
for the measurement of sea surface temperature via satellite remote sensing”, Bull. Amer. Meteor. Soc., 77, 41- 51 (1996).

29. H. H. Aumann, M. T. Chahine, D. Barron, "Sea surface temperature measurements with AIRS: RTG.SST comparison", Proc. SPIE 5151, 252-261 (2003).

30. J. W. Salisbury, and D. M. D'Aria, "Emissivity of terrestrial material in the 8-14 $\mu \mathrm{m}$ atmospheric window", Remote Sens. Environ., 42, 83-106 (1992). 